\title{
EFEITO DO TREINO LOCOMOTOR COM SUPORTE PARCIAL DE PESO CORPORAL NA VELOCIDADE DE MARCHA DE UM PACIENTE NA FASE AGUDA APÓS ACIDENTE VASCULAR CEREBRAL: ESTUDO DE CASO
}

\author{
EFFECT OF PARTIAL BODY-WEIGHT SUPPORT \\ LOCOMOTOR TRAINING ON GAIT SPEED OF A PATIENT \\ WITH ACUTE STROKE: A CASE STUDY
}

\author{
Sarah Hartel ${ }^{1}$, Daniele Rossato ${ }^{2}$, Luciano Palmeiro Rodrigues ${ }^{1,2}$
}

\begin{abstract}
RESUMO
O treino locomotor com suporte parcial de peso corporal (SPPC) é uma abordagem que tem sido utilizada na reabilitação do AVC. Entretanto, não há consenso na literatura sobre sua eficácia frente à reabilitação tradicional. Portanto, o objetivo desse estudo foi avaliar o efeito do treino com SPPC na velocidade de marcha após AVC na fase aguda. Um paciente, sexo masculino, 52 anos, foi avaliado através do Índice de Motricidade (IM) e Teste de Caminhada de 10 metros (TC10m). Recebeu, durante 5 dias, sessões de fisioterapia de 30 minutos, com 15 minutos de treino de marcha com SPPC. Apresentou um aumento de 28 pontos no IM e de mais de $50 \%$ nas velocidades de marcha normal e rápida. O resultado corrobora com estudos que evidenciam a eficácia do treino com SPPC na melhora da velocidade de marcha após AVC na fase aguda.
\end{abstract}

Palavras-chave: AVC; reabilitação; marcha

\section{ABSTRACT}

Locomotor training with partial body-weight support (PBWS) has been used for some years in stroke rehabilitation. However, there is no consensus in the literature about its effectiveness compared to conventional rehabilitation. Thus, the aim of this study was to evaluate the effect of PBWS training on gait speed after acute stroke. A 52-year-old male patient was assessed through Motricity Index (MI) and 10-meter Walk Test (10MWT). He had 30-minute physical therapy sessions, with 15-minute gait training with PBWS, for 5 days. The patient showed an increase of 28 points in $\mathrm{Ml}$ and of over $50 \%$ in normal and fast gait speeds. The results are consistent with previous studies that showed the effectiveness of PBWS training in improving gait speed in acute stroke.

Keywords: Stroke; rehabilitation; gait

Indivíduos com Acidente Vascular Cerebral (AVC) podem desenvolver incapacidades diversas ${ }^{1}$, refletindo diretamente no equilíbrio e desempenho da marcha. O comprometimento motor causa uma série de alterações em suas características espaço-temporais, dentre elas a redução da velocidade, do comprimento do passo e da passada, quando comparados a indivíduos saudáveis ${ }^{2}$.

O treino de marcha com suporte parcial de peso corporal (SPPC) tem sido utilizada há alguns anos no campo da reabilitação ${ }^{3,4}$. O SPPC oferece diminuição simétrica de peso nas extremidades inferiores, facilitando a marcha em pacientes com doenças neurológicas. Por estimular passadas mais repetitivas e rítmicas, bem como possibilitar um início precoce do treino da marcha, o SPPC favorece uma melhor recuperação da mobilidade. Além
Clin Biomed Res. 2019;39(2):175-178

1 Faculdade de Fisioterapia, Universidade Federal do Rio Grande do Sul (UFRGS). Porto Alegre, RS, Brasil.

2 Serviço de Fisioterapia, Hospital de Clínicas de Porto Alegre (HCPA). Porto Alegre, RS, Brasil.

Autor correspondente:

Sarah Hartel

sarah.hartel@gmail.com

Faculdade de Fisioterapia, Universidade Federal do Rio Grande do Sul (UFRGS) Rua Felizardo, 750.

90690-200, Porto Alegre, RS, Brasil. 
disso, por proporcionar uma descarga de peso mais simétrica nos membros inferiores, o SPPC não favorece o desenvolvimento de estratégias compensatórias e um padrão de marcha assimétrico ${ }^{5-7}$.

Apesar de já ser utilizado como um recurso adicional à terapia, ainda há uma falta de consenso na literatura sobre a real eficácia do treino de marcha com SPPC frente à reabilitação tradicional de pacientes após AVC. Sendo assim, o objetivo deste estudo foi avaliar o efeito do treino locomotor com SPPC nas velocidades de marcha normal e rápida de um indivíduo após AVC isquêmico na fase aguda.

\section{RELATO DO CASO}

Paciente do gênero masculino, 52 anos, $1,65 \mathrm{~m}$, $81 \mathrm{~kg}$, residente na cidade de Guaíba/ RS, casado, barbeiro, destro. Esteve internado na emergência do Hospital de Clínicas de Porto Alegre (HCPA), apresentando-se afásico e hemiparético à direita. Chegou ao hospital várias horas após início dos sintomas, fora do tempo hábil para realização de trombólise, sendo diagnosticado AVC isquêmico em região de mesencéfalo e ponte à esquerda. Foi classificado como nível 1 da Categoria de Deambulação Funcional (FAC), por requerer firme suporte contínuo de uma pessoa para ajudar no suporte de peso e equilíbrio. Na escala NIHSS (National Institute of Health Stroke Scale) atingiu 7 pontos, caracterizando-o como tendo um déficit moderado.

A avaliação inicial foi 11 dias após o evento, já na Unidade de AVC do HCPA. Utilizou-se o Índice de Motricidade (IM) para avaliar comprometimento de membro inferior de acordo com a força muscular do paciente para os movimentos de dorsiflexão de tornozelo, extensão de joelho e flexão de quadril do hemicorpo acometido, em sedestação à beira do leito. A pontuação pode variar de 0 (comprometimento extremamente severo) a 99 pontos (sem comprometimento) ${ }^{8}$. Também foi realizado o TC10m para quantificar a velocidade da marcha. O tempo para realização do teste foi cronometrado em segundos e a distância percorrida (10 metros) foi dividida por esse tempo, encontrando-se um valor de velocidade expresso em $\mathrm{m} / \mathrm{s}$.

No mesmo dia dos testes de avaliação, iniciaram-se as intervenções. Foram cinco dias consecutivos de atendimentos fisioterapêuticos de 30 minutos, dentre os quais 15 minutos eram destinados ao treino de marcha com SPPC, sendo os outros 15 minutos de fisioterapia convencional, já realizados como rotina na Unidade de Cuidados Especiais. A reavaliação foi realizada no dia da alta hospitalar.
Tabela 1: Parâmetros avaliados e seus resultados.

\begin{tabular}{lccc}
\hline Avaliação & Inicial & Final & $\begin{array}{c}\% \text { de } \\
\text { aumento }\end{array}$ \\
\hline $\begin{array}{l}\text { Velocidade } \\
\text { de Marcha } \\
\text { Normal }\end{array}$ & $0,21 \mathrm{~m} / \mathrm{s}$ & $0,47 \mathrm{~m} / \mathrm{s}$ & $124 \%$ \\
$\begin{array}{l}\text { Velocidade } \\
\text { de Marcha }\end{array}$ & $0,40 \mathrm{~m} / \mathrm{s}$ & $0,61 \mathrm{~m} / \mathrm{s}$ & $52,5 \%$ \\
$\begin{array}{l}\text { Rápida } \\
\text { Índice de }\end{array}$ & 44 & 72 & $\begin{array}{c}28 \text { pontos } \\
(64 \%)\end{array}$ \\
Motricidade & & & \\
\hline
\end{tabular}

Após o período de intervenção, observou-se aumento de 28 pontos no IM e aumento de mais de $100 \%$ e $50 \%$ nas velocidades normal e rápida de marcha, respectivamente (Tabela 1). Tais resultados demonstram, melhora no escore do IM, de severo para discreto, bem como um aumento nos valores de velocidades de marcha normal e rápida no TC10m, corroborando com outros estudos que demonstram a eficácia do treino locomotor com suporte parcial de peso corporal.

\section{DISCUSSÃO}

O Treino de marcha em esteira realizado com pacientes hemiparéticos comparado ao treino da marcha em solo demonstra uma melhora significativa dos parâmetros da marcha dos pacientes após o AVC, como da distância, da velocidade da marcha, do comprimento e da largura do passo ${ }^{9}$.

O último guideline para reabilitação em AVC, de 2016, traz a perda da mobilidade como uma das sequelas mais devastadoras e a reabilitação da marcha, por sua vez, como um dos principais objetivos do tratamento. Dentro desse propósito, citam uma revisão sistemática recente em que pacientes com AVC agudo (menos de 3 meses do evento) que realizam treino de marcha precoce têm uma melhor recuperação da marcha e, nesse sentido, o uso de SPPC mostrou-se mais efetivo do que o treino sem o uso desse dispositivo ${ }^{10}$.

Barbeau e Visintin ${ }^{7}$ avaliaram a velocidade de marcha de 100 pacientes com AVC subagudo, com 6 meses após o evento, divididos em dois grupos para treino de marcha em esteira: com e sem SPPC. O grupo com SPPC obteve resultados estatisticamente mais significativos do que o grupo controle, apesar de ambos terem obtido melhoras.

Sousa ${ }^{11}$ analisou os efeitos de um treino com SPPC em piso fixo na velocidade de marcha de hemiparéticos, na fase crônica do AVC, demonstrando que o treino com SPPC é uma intervenção eficaz e segura para a recuperação da marcha após AVC. Além disso, a mobilização precoce em uma fase 
aguda tem obtido resultados interessantes, como os citados por Cumming et al. ${ }^{12}$, que mostram que quanto mais intensa e precoce for a mobilização do paciente com AVC, melhor será o prognóstico de marcha e de recuperação funcional.

Veerbeek et al. ${ }^{13}$, em uma meta-análise, verificaram a evidência de diferentes abordagens fisioterapêuticas na reabilitação após AVC. Dentre as abordagens pesquisadas, o uso de SPPC para o treino de marcha em pacientes hemiparéticos, tanto na fase aguda, quanto na crônica, mostrou significante melhora na velocidade de marcha normal, apesar de não encontrar diferença significativa na velocidade rápida.

Gama et al. ${ }^{14}$ avaliaram a velocidade de marcha, dentre outros parâmetros, de 24 pacientes com AVC crônico, divididos em dois grupos, um com SPPC na esteira e outro com SPPC no solo, por 6 semanas ( $3 x$ por semana), totalizando 18 atendimentos. Ambos os grupos tiveram melhoras tanto na velocidade de marcha quanto nos demais parâmetros. Esse estudo mostra o benefício do treino de marcha com SPPC para pacientes após AVC. Estes mesmos autores, publicaram recentemente outro estudo avaliando parâmetros de marcha, o qual reforça o benefício do uso de SPPC para a melhora da marcha de pacientes com AVC, considerando que esta é uma estratégia funcional a ser adotada como intervenção no tratamento desses pacientes ${ }^{15}$.

Diferentemente dos estudos anteriormente citados, que tiveram intervenções mais duradouras ${ }^{7,11,14}$, este estudo obteve bons resultados mesmo com poucos dias de intervenção e com o paciente em fase aguda ainda durante a internação hospitalar. Este achado pode sugerir a aplicação do SPPC em unidades hospitalares ainda em fase aguda dos pacientes com AVC, preparando o paciente para o momento da alta e para dar seguimento ao tratamento fora do hospital. Embora esse seja um estudo de caso, que não permite nenhum tipo de generalização do resultado, de acordo com os achados anteriormente citados, parece evidente que a fisioterapia associada ao treino de marcha com SPPC surtiu um efeito positivo para o paciente, uma vez que esse resultado é sustentado por resultados de estudos anteriores e está corroborando com alguns dos achados da literatura.

\section{Conflitos de Interesse}

Os autores declaram não ter conflitos de interesse.

\section{REFERÊNCIAS}

1. Faria CDCM, Saliba VA, TeixeiraSalmela LF, Nadeau S. Comparação entre indivíduos hemiparéticos com e sem histórico de quedas com base nos componentes da Classificação Internacional de Funcionalidade, Incapacidade e Saúde. Fisioter Pesqui. 2010;17(3):242-7. http:// dx.doi.org/10.1590/S180929502010000300010.

2. Luvizutto GJ, Gameiro MO. Efeito da espasticidade sobre os padrões lineares de marcha em hemiparéticos. Fisioter Mov. 2011;24(4):705-12. http://dx.doi.org/10.1590/S010351502011000400015.

3. Dutra CMR, Dutra CMR, Moser ADL, Manffra EF. Treino locomotor com suporte parcial de peso corporal na reabilitação da lesão medular: revisão da literatura. Fisioter Mov. 2013;26(4):907-20. http://dx.doi.org/10.1590/S010351502013000400019 .

4. Behrman AL, Harkema SJ. Locomotor training after human spinal cord injury: a series of case studies. Phys Ther. 2000;80(7):688-700. PMid:10869131.
5. Visintin M, Barbeau H, KornerBitensky N, Mayo NE. A new approach to retrain gait in stroke patients through body weight support and treadmill stimulation. Stroke. 1998;29(6):1122-8. http://dx.doi. org/10.1161/01.STR.29.6.1122. PMid:9626282.

6. Cunha IT JR, Lim PA, Qureshy H, Henson $\mathrm{H}$, Monga T, Protas EJ. Gait outcomes after acute stroke rehabilitation with supported treadmill ambulation training: a randomized controlled pilot study. Arch Phys Med Rehabil. 2002;83(9):1258-65. http:// dx.doi.org/10.1053/apmr.2002.34267. PMid:12235606.

7. Barbeau H, Visintin M. Optimal outcomes obtained with body-weight support combined with treadmill training in stroke subjects. Arch Phys Med Rehabil. 2003;84(10):1458-65. http://dx.doi.org/10.1016/S00039993(03)00361-7. PMid:14586912.

8. Wade DT. Measurement in neurological rehabilitation. Curr Opin Neurol Neurosurg. 1992;5(5):682-6. PMid:1392142.
9. Langhammer B, Stanghelle JK. Exercise on a treadmill or walking outdoors? A randomized controlled trial comparing effectiveness of two walking exercise programmes late after stroke. Clin Rehabil. 2010;24(1):46-54. http://dx.doi. org/10.1177/0269215509343328. PMid:20026572.

10. Winstein CJ, Stein J, Arena R, Bates B, Cherney LR, Cramer SC, et al. Guidelines for adult stroke rehabilitation and recovery: a guideline for healthcare professionals from the American Heart Association/ American Stroke Association. Stroke. 2016;47(6):e98-169. http://dx.doi. org/10.1161/STR.0000000000000098. PMid:27145936.

11. Sousa CO. Estudo da marcha com suporte parcial de peso corporal em piso fixo em pacientes hemiparéticos [dissertação]. São Carlos: Universidade Federal de São Carlos; 2009.

12. Cumming TB, Thrift AG, Collier JM, Churilov L, Dewey HM, Donnan $\mathrm{GA}$, et al. Very early mobilization after stroke fast-tracks return to 
walking: further results from the phase II AVERT Randomized Controlled

Trial. Stroke. 2011;42(1):152-

8. http://dx.doi.org/10.1161/

STROKEAHA. 110.594598 .

PMid:21148439.

13. Veerbeek JM, Van Wegen E, Van Peppen R, Van Der Wees PJ,

Hendriks E, Rietberg M, et al. What is the evidence for physical therapy poststroke? A systematic review and meta-analysis. PLOS One. 2014;9(2):1-33. http://dx.doi. org/10.1371/journal.pone.0087987. PMid:24505342.

14. Gama GL, Celestino ML, Barela JA, Forrester L, Whitall J, Barela AM. Effects of gait training with body weight support on a treadmill versus overground in individuals with stroke. Arch Phys Med Rehabil.
2017;98(4):738-45. http://dx.doi. org/10.1016/j.apmr.2016.11.022. PMid:28034719.

15. Gama GL, Celestino ML, Barela JA, Barela AMF. Gait initiation and partial body weight unloading for functional improvement in poststroke individuals. Gait Posture. 2019;68:305-10. http://dx.doi. org/10.1016/j.gaitpost.2018.12.008. PMid:30553152.

Recebido: 5 mar, 2019

Aceito: 29 maio, 2019 